\title{
Overenthusiastic treatment of infertility by budding gynecologist leading to moderate OHSS
}

\author{
Krishna Dahiya, Pushpa Dahiya, Kriti Agarwal*, Shaveta Jain
}

Department of Obstetrics and Gynecology, Pandit Bhagwat Dayal Sharma Post Graduate Institute of Medical Sciences, Rohtak, Haryana, India

Received: 12 February 2017

Revised: 04 March 2017

Accepted: 08 March 2017

\section{*Correspondence:}

Dr. Kriti Agarwal,

E-mail: kriti.leo@gmail.com

Copyright: ( ) the author(s), publisher and licensee Medip Academy. This is an open-access article distributed under the terms of the Creative Commons Attribution Non-Commercial License, which permits unrestricted non-commercial use, distribution, and reproduction in any medium, provided the original work is properly cited.

\begin{abstract}
Ovarian hyperstimulation syndrome (OHSS) is a serious iatrogenic complication of luteal phase or early pregnancy, occurring in up to $5 \%$ of women undergoing in vitro fertilization (IVF) or intrauterine insemination (IUI) procedures. A 19-year-old nulligravida married for six months presented with complaint of lower abdominal pain and lump abdomen for three days. Ovulation induction was started by a private practitioner with Clomiphene citrate (50mg OD, day3 to day 7 of menstrual cycle) followed by Human Menopausal Gonadotropin (HMG) on day10, 11, 12 and Human Chorionic Gonadotropin(HCG) on day 15 of the same cycle. Her symptoms started 14 days following injection of HCG. Abdominal examination demonstrated pelvic abdominal mass up to the level of umbilicus corresponding to 24 weeks' size, tense, non-tender, mobile and of cystic consistency. Ultrasonograph of abdomen revealed uterus of normal size, endometrial thickness-20mm, right ovary of size $81 \times 95 \times 119 \mathrm{~mm}$ with multiple cysts and left ovary of size $65 \times 61 \times 66 \mathrm{~mm}$ with mild ascites. She was managed conservatively with bed rest, abdominal girth measurement, oral analgesics, intake output charting with plenty oral fluids and gentle leg exercises. Within two weeks of admission, pain was relieved and serial hematological and biochemical parameters remained normal. Repeat ultrasonography confirmed the presence of intrauterine live pregnancy and she was discharged. All gynecologists must know when, how and where to treat cases of infertility in order to prevent OHSS and its complications like renal failure, ARDS,thromboembolism,ovarian torsion and intra peritoneal hemorrhage.
\end{abstract}

Keywords: Infertility, Moderate, Ovarian hyperstimulation syndrome

\section{INTRODUCTION}

Ovarian hyperstimulation syndrome (OHSS) is a serious iatrogenic complication of luteal phase or early pregnancy, occurring in up to $5 \%$ of women undergoing in vitro fertilization (IVF) or intrauterine insemination (IUI) procedures. ${ }^{1}$ Its occurrence is dependent on the administration of human chorionic gonadotropin (HCG) after an exaggerated ovarian response to gonadotropin stimulation and is only rarely observed after clomiphene citrate therapy or spontaneous ovulation., ${ }^{2,3}$ Cystic enlargement of ovaries with fluid shift from intravascular to third space compartments due to increased vascular permeability and ovarian neoangiogenesis is the main pathogenesis of the syndrome. Studies have shown serum vascular endothelial growth factor (VEGF) levels to correlate with the severity of OHSS. ${ }^{4}$ It results in varied clinical presentations including ascites, pleural and pericardial effusion, hemoconcentration, oliguria, electrolyte imbalance and hypercoagulability.

Several risk factors have been reported to increase its incidence, including polycystic ovary syndrome, high serum estradiol (>2500 pg/ml), HCG supplementation of the luteal phase, women $<35$ years, and the occurrence of conception in the cycle of treatment. ${ }^{5}$ We report a case of 
moderate OHSS occurring in first cycle of treatment in a young female due to overenthusiastic treatment by budding gynecologists within six months of marriage.

\section{CASE REPORT}

A 19-year-old nulligravida married for six months presented with complaint of lower abdominal pain and lump abdomen for three days. Ovulation induction was started by a private practitioner as she was eager to conceive, with Clomiphene citrate $(50 \mathrm{mg}$ OD, day3 to day 7 of menstrual cycle) followed by human menopausal gonadotropin (HMG) on day10, 11, 12 and human chorionic gonadotropin (HCG) on day 15 of the same cycle. Her symptoms started two weeks following injection of HCG.

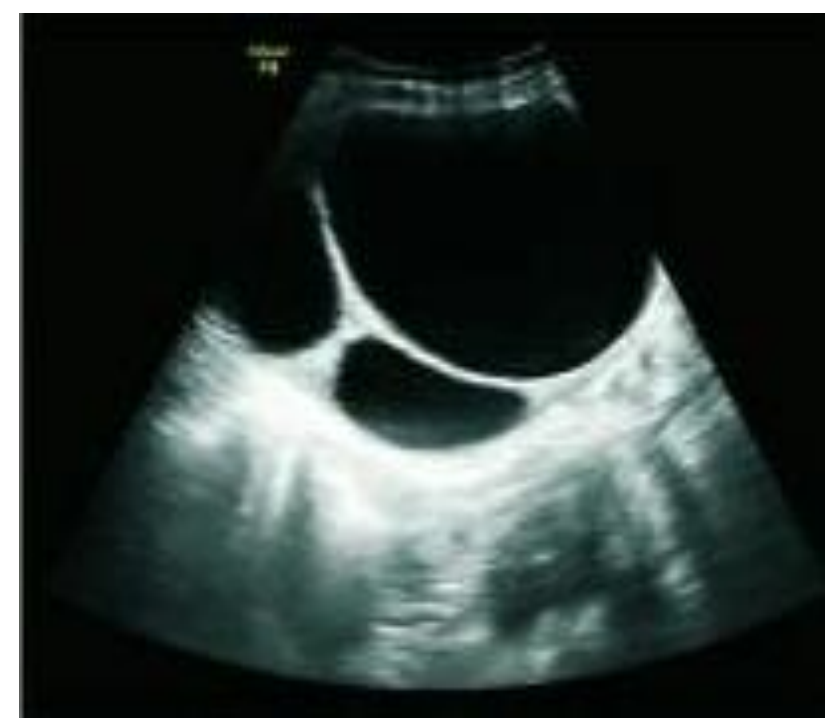

Ultrasound showing right ovary of size $81 \times 95 \times 119 \mathrm{~mm}$ with multiple cysts

Figure 1: Right ovary.

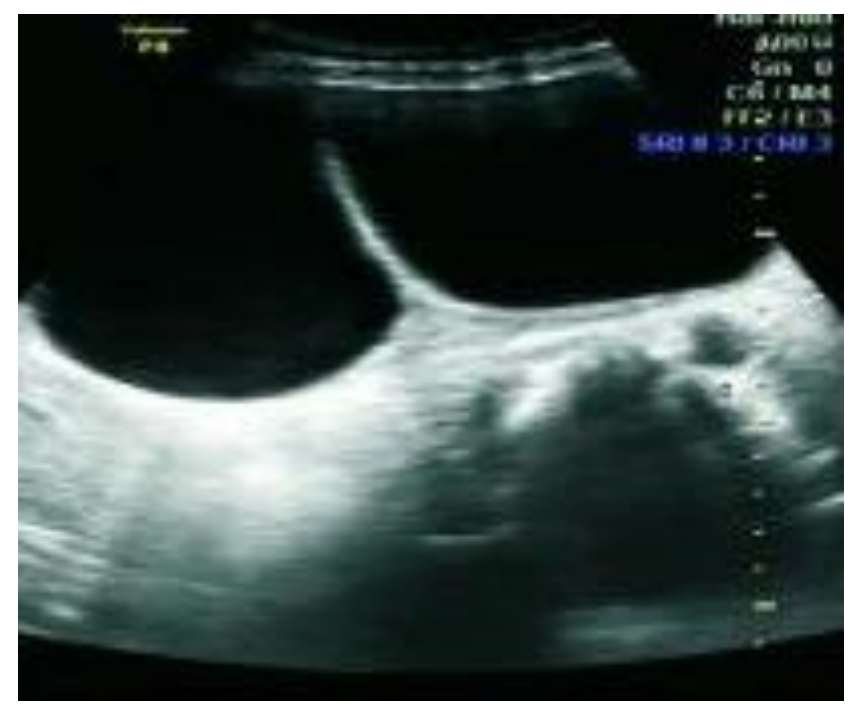

Ultrasound showing left ovary of size $65 \times 61 \times 66 \mathrm{~mm}$

Figure 2: Left ovary.
On examination, BMI-18.9, Pulse-82/min, BP-100/60 $\mathrm{mm} \mathrm{Hg}$, respiratory rate-14 breaths per minute, afebrile. General examination was normal. Abdominal examination demonstrated pelvic abdominal mass up to the level of umbilicus corresponding to 24 weeks' size, tense, non-tender, mobile and of cystic consistency. In view of suspected OHSS, pelvic examination was not done. Examination of other systems was unremarkable. Ultrasonography revealed uterus of normal size, endometrial thickness-20mm, right ovary of size $81 \times 95 \times 119 \mathrm{~mm}$ with multiple cysts (Figure 1) and left ovary of size $65 \times 61 \times 66 \mathrm{~mm}$ with mild ascites (Figure 2). Urine pregnancy test was negative at admission. Hematological investigations were as follows: $\mathrm{Hb} 11.2$ gm/dl, PCV 40\%, total leukocyte count-6,800 and platelet-2 lakhs. Renal function test, liver function tests, thyroid and coagulation profile, serum electrolytes were normal. She was managed conservatively with bed rest, abdominal girth measurement, oral analgesics, intake output charting with plenty oral fluids and gentle leg exercises. Within two weeks of admission, pain was relieved and serial hematological and biochemical parameters remained normal. Repeat ultrasound confirmed the presence of early intrauterine live pregnancy and she was discharged.

\section{DISCUSSION}

OHSS can be early or late depending upon time of onset of symptoms. Early occurs within 10 days of injection of HCG and is due to exaggerated ovarian response to gonadotropin stimulation, whereas late OHSS occurs more than 10 days after HCG administration and is related to secretion of placental HCG. Spontaneous OHSS is likely to occur at 8-14 weeks of gestation, while iatrogenic OHSS occurs earlier at 3-8 weeks of gestation. ${ }^{6}$ OHSS is divided into four grades (mild, moderate, severe, and critical) according to the classification modified by Mathur et al. ${ }^{3}$

Mild ovarian hyperstimulation occurs in 8-23 percent of stimulated cycles, moderate forms in $<1-7$ percent and severe forms in 0.5 percent of stimulated cycles. ${ }^{7}$ Onset of abdominal pain two weeks after receiving HCG injection and ultrasonographic evidence of ascites with ovarian size of $12 \mathrm{~cm}$ categorized our case as moderate OHSS of late onset type. Late onset OHSS is more severe than early onset OHSS. ${ }^{8}$ Certain vasoactive substances such as vascular endothelial growth factor (VEGF), cytokines (IL-2, IL-6, and IL-8), tumor necrosis factoralpha (TNF-alpha), and the ovarian renin-angiotensin system, which are activated by gonadotropin, can lead to increased vascular permeability and extravascular fluid accumulation in OHSS which is responsible for development of ascites, pleural effusions, edema, and hemoconcentration. ${ }^{9}$ In spontaneous OHSS extravascular fluid retention may not occur and usually a hemodilution rather than hemoconcentration is seen. ${ }^{10}$ Presentation may vary from gastrointestinal upset and abdominal distension in mild cases to dyspnea and hypovolemia in severe 
cases. Critical cases include hydrothorax, severe thromoboembolism and acute respiratory distress syndrome, which require admission to Intensive Care Unit. Symptoms generally resolve in 1-2 weeks but will be more severe and persist longer if pregnancy occurs, due to HCG acting on corpus luteum in the ovaries. Typically in severe OHSS with developing pregnancy the duration does not exceed first trimester. In our case, the symptoms resolved in first trimester within two weeks of onset, despite coexisting pregnancy. Mild and moderate OHSS can be managed on outpatient basis by limiting activity, daily weight measurement, abdominal girth charting, intake output charting, oral anti-emetics and analgesics, laboratory monitoring of hematocrit, electrolytes, renal and liver function tests and coagulation profile. Severe OHSS is treated with intravenous fluids, low molecular weight heparin, abdominal paracentesis for symptomatic relief of abdominal discomfort or dyspnea caused by pronounced ascites. Surgery is required for ovarian torsion or rupture. Preventive measures include cycle cancellation, coasting, using lower dose of $\mathrm{HCG}$ injection, recombinant Lutenising Hormone, cabergoline in last week of stimulation till 1-3 week after HCG stimulation, cryopreservation, in-vitro maturation of immature oocytes, VEGF receptor antagonist.

\section{CONCLUSION}

OHSS can lead to complications like renal failure, liver failure, thromboembolism, ARDS, ovarian torsion and rupture with intra-peritoneal haemorrhage. Hence, all gynecologists must know when, how and where to treat cases of infertility. In our case, OHSS was definitely preventable, as ovulation induction was not indicated in such a short period of marriage, especially with gonadotropins and HCG injection.

Funding: No funding sources

Conflict of interest: None declared

Ethical approval: Not required

\section{REFERENCES}

1. Neulen J, Yan Z, Raczek S, Weindel K, Keck C, Weich HA, et al. Human chorionic gonadotropindependent expression of vascular endothelial growth factor/vascular permeability factor in human granulosa cells: importance in ovarian hyperstimulation syndrome. J Clin Endocrinol Metab. 1995;80:1967-71.

2. Yildizhan R, Adali E, Kolusari A, Kurdoglu M, Ozgokce C, Adali F. Ovarian Hyperstimulation Syndrome with pleural effusion: a case report. Cases J. 2008;1:323.

3. Marthur R, Kailasam C, Jenkins J. Review of the evidence base strategies to prevent ovarian hyperstimulation syndrome. Hum Fertil. 2007;10:7585.

4. Geva E, Laffe RE. Role of vascular endothelial growth factor in ovarian physiology and pathology. Fertil Steril. 2007;74:429-38.

5. Haning RV, Austin CW, Carlson IH, Kuzma DL, Shapiro SS, Zweibel WJ. Plasma estradiol is superior to ultrasound and urinary estriol glucronide as a predictor of ovarian hyperstimulation during induction of ovulation with menotropins. Fertil Steril. 1983;40(1):31-6.

6. Smits G, Olatunbosun O, Delbaere A. Ovarian hyperstimulation syndrome due to a mutation in the follicle stimulating hormonereceptor. N Eng J Med. 2003;349:760-6.

7. Navot D, Bergh PA, Laufer N. Ovarian hyperstimulation syndrome in novel reproductive technologies: prevention and treatment. Fertil Sertil. 1992;58:249-61.

8. Mathur RS, Akande AV, Keay SD, Hunt LP, Jenkins JM. Distinction between early and late ovarian hyperstimulation syndrome. Fertil Steril. 2000;73:901-7.

9. Elchalal U, Schenker JG. The pathophysiology of ovarian hyperstimulation syndrome- views and ideas. Hum Reprod. 1997;12:1129-37.

10. Smisha S and Sridev B. Case report on spontaneous ovarian hyperstimulation syndrome following natural conception and associated with primary hypothyroidism. J Hum Reprod Sci. 2013;6(2):158-61.

Cite this article as: Dahiya $\mathrm{K}$, Dahiya $\mathrm{P}$, Agarwal K, Jain S. Overenthusiastic treatment of infertility by budding gynecologist leading to moderate OHSS. Int J Reprod Contracept Obstet Gynecol 2017;6:1686-8. 